J3eA, Journal sur l'enseignement des sciences et technologies de l'information et des systèmes, Volume 3, Hors-Série 1, 17 (2004)

DOI : http://dx.doi.org/10.1051/bib-j3ea:2004617

(C) EDP Sciences, 2004

Système visuo-tactile d'aide à la mobilité indépendante des déficients visuels dans des environnements 3D non -coopérants

R. Velázquez ${ }^{1}$, E. Pissaloux ${ }^{1}$, J. Szewczyk ${ }^{1}$ et M. Hafez ${ }^{2}$

${ }^{1}$ Laboratoire de Robotique de Paris

CNRS FRE 2507

18 route du panorama

BP 61

F-92265 Fontenay-aux-Roses, France

${ }^{2}$ CEA/LIST

18 route du panorama

BP 61

F-92265 Fontenay-aux-Roses, France

velazquez@robot.jussieu.fr 


\title{
Système Visuo-Tactile d'Aide à la Mobilité Indépendante des Déficients Visuels dans des Environnements 3D Non-Coopérants
}

\author{
Ramiro Velázquez ${ }^{1}$, Edwige Pissaloux ${ }^{1}$, Jérôme Szewczyk ${ }^{1}$ et Moustapha Hafez ${ }^{2}$ \\ ${ }^{1}$ Laboratoire de Robotique de Paris, CNRS FRE 2507 \\ 18 Route du Panorama, BP 61, 92265 Fontenay aux Roses, France \\ ${ }^{2}$ CEA/LIST, 18 Route du Panorama, BP 61, 92265 Fontenay aux Roses, France \\ velazquez@robot.jussieu.fr
}

\begin{abstract}
Résumé - La navigation et la mobilité sont considérées comme les problèmes les plus importants chez les personnes souffrant d'une déficience visuelle. Cet article présente brièvement le projet de "Lunettes Intelligentes ", une aide aux déplacements indépendants et en sécurité de déficients visuels. Ce projet, en cours de développement au Laboratoire de Robotique de Paris (LRP) et au CEA/LIST, vise à concevoir et à réaliser un système qui fournirait aux utilisateurs (les aveugles, les mal-voyants et les personnes âgées) une information sur la disposition spatiale des obstacles localisés dans leur environnement immédiat. Le prototype se compose d'un système de vision stéréoscopique et d'une surface à stimulation tactile ; le système de vision construit la carte des obstacles (statiques et dynamiques) qui sera affichée sur la surface à stimulation tactile balayée par l'utilisateur. Le concept fondamental de cette recherche - le concept d'une nouvelle interface homme-machine visuo-tactile reprogrammable - peut être facilement transposé à d'autres applications où la perception visuelle est limitée, voire absente mais essentielle pour exécuter une tâche, comme la robotique mobile, la robotique médicale, les environnements virtuels, les jeux...
\end{abstract}

Mots clés : AEM (aides électroniques à la mobilité), carte d'obstacles, stéréovision, surface à stimulation tactile, taxel.

\section{INTRODUCTION}

Selon le rapport 2002 de l'Organisation Mondiale de la Santé (OMS), il y a plus de 180 millions de mal-voyants à travers le monde, dont 45 millions sont des aveugles; plus de 8 millions d'entre eux habitent en Europe, et il est à prévoir que ce chiffre doublera dans les vingt années à venir car la société européenne vieillit [Oms02].

L'assistance aux déficients visuels est donc un problème très important en termes de santé publique et est responsable de dépenses importantes de sécurité sociale. Le déficient visuel et sa famille sont confrontés dans leur vie quotidienne à des contraintes socioéconomiques majeures. Aussi, les personnes âgées mal-voyantes ou non-voyantes et les aveugles souhaitent vivre d'une façon plus indépendante et mieux intégrés à la société moderne.

Cependant, leur permettre d'acquérir les gestes fondamentaux de la vie, nécessite l'intervention de plusieurs spécialistes : instructeur psychomoteur, spécialiste de la réhabilitation, de l'orientation, etc. Il s'agit des spécialistes médicaux et d'assistance entraînant des coûts très élevés pour le handicapé. Les financements disponibles et le personnel qualifié existant ne couvrent pas les besoins réels de la population. Pour ces raisons, il est important de concevoir et de réaliser des aides technologiques nécessaires à la compensation de la déficience visuelle.

La technologie de nos jours propose des solutions dans tous les domaines de la médecine et de la réhabilitation. Une interface haptique adéquate pourrait représenter une solution potentielle au problème de la mobilité chez les aveugles et les mal-voyants.

Le projet «Lunettes Intelligentes» se place dans ce contexte. Le système proposé offre une solution de mobilité et de détection d'obstacles pour permettre la locomotion indépendante en toute sécurité des déficients visuels.

La suite de cette communication propose un bref état de l'art sur les aides électroniques à la mobilité (AEM) (\$2), puis introduit le concept de l'interface visuo-tactile (IVT) (§3) et donne un aperçu d'une matérialisation d'IVT sous forme de «Lunettes intelligentes» (LI). La conclusion renferme l'état du projet (IVT-LI) et les futures directions de recherche.

\section{AEM : UN ETAT DE L’ART}

La recherche sur les systèmes d'aide à la perception de scènes pour les déficients visuels a exploré essentiellement deux domaines : la transmission de l'information et l'assistance à la mobilité.

La transmission de l'information comprend la lecture, la reconnaissance des caractères et l'affichage graphique des scènes 2D et 3D. Le code Braille, introduit au 19ème siècle par Louis Braille, est devenu le standard de lecture ${ }^{1}$ le plus populaire chez les déficients visuels du monde entier.

La transmission graphique s'est appuyée essentiellement sur des interfaces tout d'abord en relief, puis tactiles. Depuis 1960, plusieurs projets ont visé le développement d'une interface qui permettrait une représentation précise de l'information. Les prototypes les plus connues sont l'Optacon [Bli70], le TVSS [Bac69], le Dot Matrix [Met], le VDT-TVSS [Tho00], ainsi que les nombreux projets de recherche développés comme ceux de Shinohara [Shi98], Howe [How93] et Srinivasan [Sri97].

Cependant, il n'existe pas encore de système ou de prototype constituant une réelle avancée par rapport aux aides classiques aux déplacements. En effet, le problème de la mobilité de l'humain ne se réduit pas à un simple déplacement d'un point de l'espace à l'autre (comme cela est le cas de robots). Confronter un déficient visuel à la mobilité implique impérativement d'avoir l'information

\footnotetext{
${ }^{1}$ C'est aussi un code pour d'autres domaines comme la musique.
} 
spatiale quasi globale de l'environnement le plus proche, d'avoir l'information de l'orientation et de localisation des obstacles, et, peut être avant tout, de percevoir l'espace. En effet, la perception quasi globale de l'espace est le facteur inhérent de l'autonomie et la sécurité de nos déplacements [Ber97].

Les aides proposées jusqu'à présent, connues sous le nom d'aides à la mobilité (AM), essayent de prendre totalement en charge l'utilisateur lui laissant peu de possibilité de percevoir, de comprendre et d'apprendre l'environnement dans lequel il évolue. Les AM les plus utilisées sont la canne blanche et le chien-guide. La canne blanche, un dispositif mécanique très simple, est un outil effectif pour la détection des obstacles rigides au niveau du sol ; elle est moins efficace pour la détection des surfaces à déclivité variable et peu efficace pour la détection des trous. Elle est légère et portable, et son prix est abordable. Cependant, sa capacité de "perception" de l'environnement est limitée et son apprentissage est long [Nfb]. Les chiens guide sont des aides efficaces lors de déplacements dans un environnement connu. En revanche, un chien n'est pas capable de prendre une décision en cas de situation non apprise à l'entraînement [Pis03]. En outre, un chien exige des soins constants et leur prix est loin d'être accessible.

Depuis les années 60, la technologie a permis de développer des aides électroniques à la mobilité (AEM). Parmi ces dispositifs les plus connus sont la Canne Laser C-5 [Ben73], l'Aide Sonic Binaurale [Kay74], le détecteur des obstacles de Nottingham [Bis80], le capteur de Mowat [Wor], la Canne Guide [Ulr01], le NavBelt [Bor90], le système vOICe [Mei98] et le Télétact [Far02]. Bien qu'il existe une très grande variété d'AEM, peu de ces dispositifs sont réellement adoptés par les malvoyants ou les aveugles. La faible assistance effective apportée par ces dispositifs lors des déplacements, et la complexité de leur interface semblent être les principales raisons de la non appropriation de ces outils. On peut donc conclure qu'il est urgent de reétudier le problème de conception des AEM afin qu'ils assistent efficacement et en sécurité, les déplacements indépendants des déficients visuels. Le projet «LI » vise à concevoir et réaliser une telle aide.

\section{CONCEPT DES LUNETTES INTELLIGENTES}

Nous envisageons d'améliorer la performance des AEM existantes en proposant une nouvelle technique de navigation à travers la plate-forme « LI ». Ce projet tente de répondre aux critères de sécurité et d'indépendance du déficient visuel en localisant certaines classes prédéfinies d'obstacles dans son environnement immédiat. Ainsi en délimitant les zones non accessibles, on indiquera les zones de déplacements possibles.

On s'attend à identifier quatre classes d'obstacles : obstacles statiques situés au niveau du sol ou au-dessus du sol (c.-à-d. les objets suspendus), obstacles statiques situés au-dessous du niveau du sol (par exemple des trous) et obstacles dynamiques.

Le système de «LI» (Figure 1) se compose de deux sous-systèmes : le sous-système de vision et le sous- système à stimulation tactile. Le sous-système de vision sert à l'analyse, l'optimisation et la retranscription d'informations sur les classes identifiées d'obstacles se trouvant dans l'environnement 3D le plus proche du déficient visuel. Deux images stéréo sont à la base du processus de délimitation des obstacles. Différents algorithmes de vision permettent de construire une représentation adéquate de l'environnement $3 \mathrm{D}$ et de la retranscrire en une représentation tactile ; cette dernière est affichée sur l'interface à stimulation tactile (de taille d'une disquette) pour une exploration rapide avec la main [Pis00].

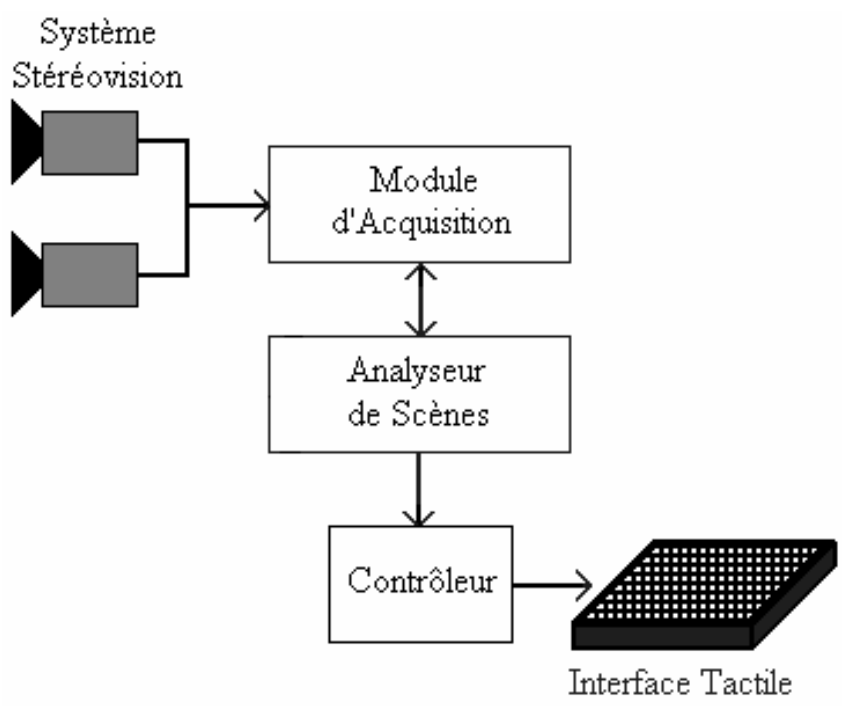

Fig.1. Architecture générale du système des Lunettes Intelligentes.

La figure 2 résume le mode opératoire du système « LI ». Remarquons que la carte tactile délimitera les zones à partir du contour des objets les plus avancés spatiallement et projetés sur une surface $2 \mathrm{D}$. Il semble que notre cerveau procède ainsi [Ber97]. Soulignons que ce mode d'exploitation de l'information est assez intuitif, et semble correspondre au critère de «design for all », c.-à-d. de conception d'interfaces homme-machine pouvant être facilement comprises tant par les déficients visuels que par les aveugles ${ }^{2}$.

\section{A. Système Stéréo Vision}

On sait que la projection d'une scène $3 \mathrm{D}$ sur le plan d'une image, conduit à une représentation 2D de la scène dans laquelle l'information de profondeur est perdue. Cependant, cette information peut être reconstituée à l'aide d'images stéréoscopiques. Le système de vision se compose donc de deux caméras solidaires et pré-calibrées fournissant des images stéréo.

\footnotetext{
2 La représentation de l'environnement immédiat d'un aveugle par les contours des objets est un mode privilégié de l'apprentissage de l'organisation de notre monde par des jeunes aveugles à l'INJA (Institut National des Jeunes Aveugles) à Paris.
} 


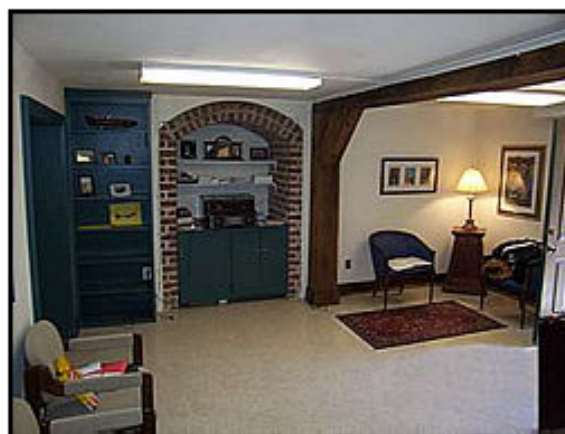

a)

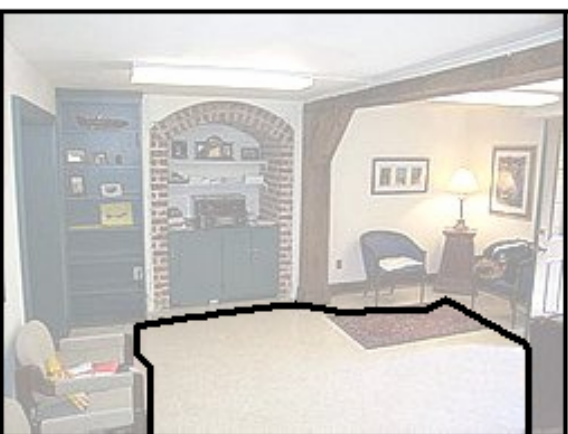

b)

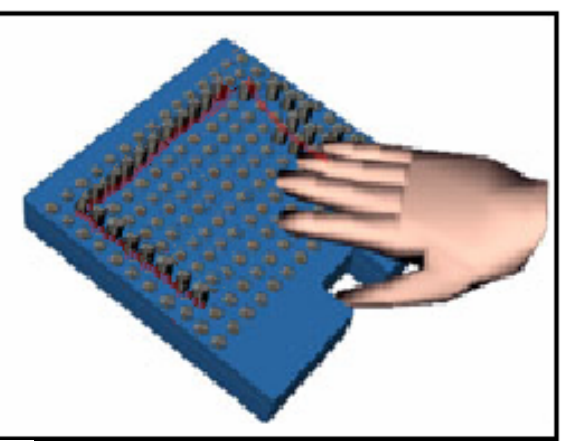

c)

Fig. 2. Résultats attendus: a) L'image mosaïque obtenue avec le système stéréovision représentant l'environnement immédiat, b) les obstacles dans la scène sont identifiés par l'intermédiaire des algorithmes de vision et c) les obstacles sont affichés sur l'interface tactile, l'identification de leur position permet alors de déduire la voie à suivre.

L'information de profondeur est essentielle pour la détection des obstacles (et donc pour la délimitation des zones); elle permet de localiser les obstacles dans la scène. La figure $3 \mathrm{a}$ rappelle un modèle mathématique d'un système de stéréo vision épipolaire rectifié [Fu87], tandis que la figure $3 \mathrm{~b}$ montre le prototype physique expérimental en cours de conception et de réalisation au LRP et au CEA. En utilisant deux caméras, il est possible d'obtenir deux images de deux points de vue séparés des objets dans la même scène (figures $3 \mathrm{c}$ et $3 \mathrm{~d}$ ). Pour obtenir la profondeur d'un point de la scène, les deux points homologues qui lui correspondent doivent être trouvés dans les deux images et appariés.

Les deux caméras (CCD Pacific - CSB465C) de notre prototype sont identiques (de même focale, de mêmes caractéristiques photométriques) et leur système de coordonnées, figé à l'aide d'un support rigide, est parfaitement aligné (système épipolaire). La longueur de la ligne de base $\mathrm{B}$, reliant les centres de projection des deux caméras, est adaptative; elle correspond à la variation de distance entre les yeux humains $(10-20 \mathrm{~cm})$. De même, l'angle entre les deux caméras est réglable, mais pour les images rectifiées, les axes optiques de deux caméras sont parallèles.

\section{B. Analyseur de Scènes}

Ce module traite la scène sous forme de deux images stéréo épipolaires. Il calcule deux fonctions principales : la détection des obstacles et leur localisation dans la scène (et donc par rapport à l'utilisateur).

Plusieurs algorithmes de détection des obstacles peuvent être mis en oeuvre. Nous nous dirigeons vers la mise en correspondance des images stéréo épipolaires (stereo image matching) à l'aide de l'algorithme de la programmation dynamique. Grâce à un circuit dédié, cette méthode met en correspondance des pixels homologues de deux lignes épipolaires de $\mathrm{N}$ pixels en temps $0(\mathrm{~N})$ [Pct00]. Afin d'augmenter la robustesse de l'appariement et de réduire sa complexité temporelle, chaque pixel est caractérisé par un ensemble de valeurs (encodées dans un vecteur attaché au pixel) : le niveau de gris moyen dans un voisinage $3 \times 3$, le gradient local de luminosité et la vitesse de variation de luminosité (ou Laplacien) [Pis00]. Ces caractéristiques sont utilisées pour lever les ambiguïtés éventuelles entre les points candidats à l'appariement. Les pixels candidats à l'appariement sont homologues seulement si leurs vecteurs sont égaux ou équivalents. Une fois appariés, les pixels homologues permettront d'obtenir la profondeur du pixel de la scène via la triangulation.

L'exécution en temps réel du traitement entier est une contrainte très forte de notre application. Notre définition du temps réel est basée sur la vitesse de marche régulière d'une personne $(3.6 \mathrm{~km} / \mathrm{h}$ ou $1 \mathrm{~m} / \mathrm{sec})$. Ainsi, les caméras et l'analyseur d'images devront fournir des informations sur la scène à fréquence pertinente qui reste à déterminer.

\section{Interface Tactile}

L'objectif fondamental du projet de «Lunettes Intelligentes » est de concevoir et de réaliser une interface à stimulation tactile qui restitue avec une précision choisie l'information provenant de l'extérieur (l'information fournie par le système de vision stéréoscopique) pour la transmettre à l'utilisateur final (aveugles, personnes âgées et déficients visuels).

\section{C.1. Surface de Stimulation Tactile}

L'interface de stimulation tactile se composera d'une matrice $2 \mathrm{D}$ dont le relief sera commandé par actionneurs mécaniques. L'interface affichera de cette façon l'information sur les obstacles statiques et dynamiques situés dans l'environnement proche de l'utilisateur (3 à 4 mètres devant lui, les scènes urbaines de base: les croisements de rues).

L'interaction tactile entre l'utilisateur et l'interface est l'un de nos soucis principaux. L'acceptation (l'appropriation) par les utilisateurs dépendra principalement de la nature de l'interface tactile ; si sa manipulation est suffisamment aisée et fiable, le prototype sera évidemment plus facilement adopté. 

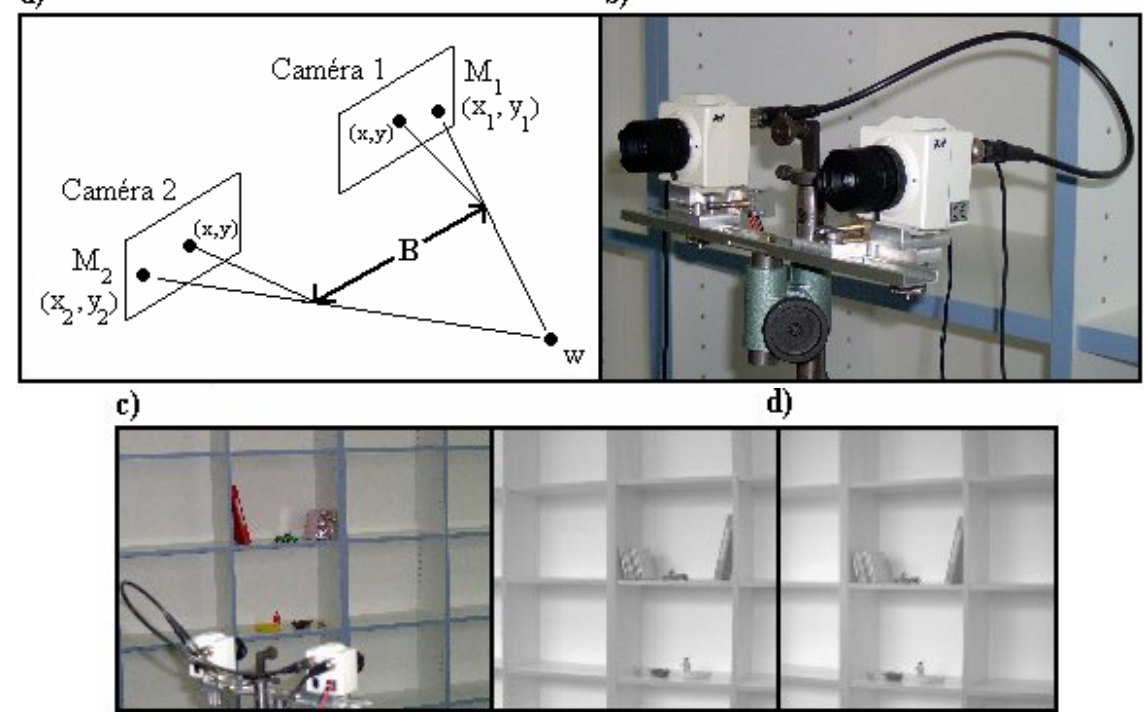

Figure 3. a) Modèle typique du processus stéréo. Le point w est enregistré par la caméra 1 comme le point $\mathrm{M}_{1}$ de coordonnées ( $x_{1}$, $y_{1}$ ) et par la caméra 2 comme le point $\mathrm{M}_{2}$ de coordonnées $\left(x_{2}, y_{2}\right)$, tous les deux référencés au centre de l'image. La ligne qui relie les centres des objectifs s'appelle la ligne de base (baseline) B. L'objectif est de trouver les coordonnées $(X, Y, Z)$ du point w. b) Le système stéréo expérimental. c) Une scène d'expérimentation quelle conque d) Sa représentation stéréo (image gauche et droit).

Afin de réaliser une interface tactile optimale, nous considérons les critères globaux de conception suivants : la facilité d'usage, la portabilité et la faible consommation d'énergie.

1. Le système tactile doit être facile à manier et exiger peu ou pas d'apprentissage spécifique. Ceci implique que l'utilisateur doit pouvoir l'employer de manière intuitive.

2. L'interface devrait être portable, c.-à-d. que le système «LI» doit être léger et de petites dimensions (3.5 pouces). Une petite surface tactile permettra un balayage rapide. Ceci est essentiel afin de pouvoir prendre des décisions rapides au moment de déplacements. Il est également important que le dispositif soit peu invasif pour l'environnement et très discret (facteurs essentiels pour les aveugles et personnes âgées).

3. Le système doit présenter une faible consommation d'énergie. La source d'énergie doit être dimensionnée pour fournir de l'énergie au système de stimulation tactile pour la durée d'un déplacement à marche régulière, et en plus rester légère afin d'être intégrée au prototype portable (comprenant aussi les caméras).

\section{C.2. Taxel}

La surface à stimulation tactile est une matrice (2D) d'actionneurs (taxels) permettant de matérialiser les différentes classes d'obstacle. Les différentes technologies de réalisation d'actionneur tactile (taxel) incluent les technologies électromagnétique et piézoélectrique, les alliages à mémoire de forme (AMF), le pneumatique et les fluides électrorhéologiques [Ben02]. Les caractéristiques et les propriétés de divers matériaux classiques et intelligents sont actuellement en cours d'évaluation en vue du développement de l'interface. La technologie retenue devra respecter les contraintes suivantes : encombrement maximal de l'actionneur imposé par les dimensions des contacts créant le relief, rigidité suffisante de ces contacts, capacité d'intégration, temps de réponse de l'ordre de $1 \mathrm{sec}$. et précision élevée.

1. Dimensions des contacts créant le relief : Plusieurs études physiologiques et du sens du toucher ont été faites afin de trouver une stimulation efficace des récepteurs de la peau humaine [Shi93], [Ber97]. Les résultats prouvent que les sensations perçues lors d'un contact mécanique passent principalement par les corpuscules ou terminaisons de Meissner et de Pacini, aussi appelés mécanorécepteurs. La densité la plus élevée de mécanorécepteurs se trouve dans la langue et au bout des doigts. A ces deux endroits, les mécanorécepteurs humains sont séparés d'une distance de 2.6 à $3 \mathrm{~mm}$ et sont disposés de façon régulière (en matrice), à 0.7 à $1.5 \mathrm{~mm}$ sous la surface de la peau [Mos92]. Ainsi, pour bien stimuler les mécanorécepteurs du doigt, la distance entre 2 contacts de notre interface et par conséquent leur diamètre maximum doit être de $3 \mathrm{~mm}$ et doit produire une hauteur de relief idéalement de $1.5 \mathrm{~mm}$.

2. Rigidité des contacts: Chaque actionneur doit pouvoir résister à la force de contact des doigts ou des paumes pendant le processus exploratoire (l'analyse de l'affichage tactile). Plusieurs études sur la force de contact entre le doigt et la surface d'exploration indiquent qu'une force de 2,3 $\mathrm{N}$ s'applique en exploration normale (dans notre cas : la surface sera effleurée) tandis que $4,4 \mathrm{~N}$ est une force de contact intentionnellement violente [Led90].

3. Capacité d'intégration: Tous les actionneurs et leur interface de contrôle doivent être optimisés spatialement. Cela conduira à un système portable et de faible poids.

4. Temps de réponse : L'actionneur doit avoir un temps de réponse approprié afin de fournir un retour tactile qui correspond à la dynamique de l'information visuelle. Il est évident que le retour tactile ne sera efficace que s'il est 
modifié à la vitesse de mise à jour de l'information visuelle.

5. Précision: Le taux de correspondance entre le nombre de pixels de l'image et un taxel définit la précision de notre système. La précision du système sera en rapport avec les 2 objectifs: la capacité d'intégration et le temps de réponse des actionneurs.

L'utilisateur pourra également obtenir des informations sur la classe de l'obstacle affiché sur l'interface en touchant la tête du taxel. Un taxel de coordonnées $(\mathrm{x}, \mathrm{y})$ aura 2 formes possibles (pleine et pointue) et deux états possibles (statique et vibratoire) permettant de coder les quatre classes d'obstacles : pas d'obstacles, les obstacles statiques localisés sur/au-dessus de la surface de référence, les obstacles statiques localisés sous la surface de référence et les obstacles dynamiques. Le tableau 1 met en correspondance les différentes configurations possibles d'un taxel en fonction de la classe d'obstacle détecté.

\begin{tabular}{|l|l|}
\hline \multicolumn{1}{|c|}{ Classes d'obstacle } & Type et forme du taxel \\
\hline Pas d'obstacle & Non activé (non sorti) \\
\hline $\begin{array}{l}\text { Obstacle statique, sur/au- } \\
\text { dessus la surface de référence }\end{array}$ & Sorti, plein, statique \\
\hline $\begin{array}{l}\text { Obstacle statique, sous la } \\
\text { surface de référence }\end{array}$ & Sorti, pointu, statique \\
\hline Obstacle dynamique & Sorti, plein, vibration \\
\hline
\end{tabular}

Table.1 Codage : Taxel - Obstacle.

On notera qu'il est important de préserver une certaine simplicité du mode d'affichage des obstacles. Ainsi, on se contentera ici des quatre configurations de taxel mentionnées ci-dessus. Ceci permettra d'éviter la saturation d'information et de provoquer la confusion de l'utilisateur, aspects qui peuvent affecter directement l'acceptation du prototype.

\section{CONCLUSION}

Cet article a présenté un système appelé «Lunettes Intelligentes », une aide à la mobilité et à la navigation indépendante et en sécurité des personnes aveugles ou malvoyantes dans un environnement non coopérant. Ce système permet la détection des obstacles tant statiques que dynamiques. Il associe un système de vision stéréo épipolaire (analyse de la scène, détection et localisation d'obstacles) et d'une surface à stimulation tactile (l'affichage des obstacles) travaillant en temps réel. La projection 2D d'objets 3D est une stratégie de perception $\mathrm{du}$ monde enseignée aux jeunes aveugles. $\mathrm{Vu}$ la représentation intuitive des obstacles sur la tablette tactile, les «Lunettes Intelligentes» constituent une interface correspondant bien au critère de « design for all ».

Deux domaines principaux de recherche composent ce projet : système de vision et interface tactile.

Actuellement nous travaillons ces deux aspects du problème :
1- Recherche de nouveaux algorithmes (d'inspiration biologique de préférence) pour l'appariement d'images stéréo épipolaires (scènes urbaines et croisement de rues principalement) et la reconstruction de la profondeur.

2- Conception et réalisation, à l'aide de technologies de pointe, d'une surface complète de stimulation tactile (32x32 taxels au moins).

Les Lunettes Intelligentes sont un exemple type d'AEM non-invasive, tant pour l'utilisateur que pour l'environnement. Ils introduisent le concept de (télé-) navigation et (télé-)détection d'obstacles. Ce principe de navigation peut être facilement transféré à d'autres domaines comme la robotique mobile, la réalité virtuelle, les jeux, l'aide à la conduite de voiture, ...

Par ailleurs, ce projet fait partie d'une plus vaste plateforme expérimentale pour les études en physiologie de la perception (projet HuPer du programme Robea du CNRS, collaborations: LRP, CEA/LIST, Collège de France, LPPA). Cette plate-forme a pour but d'approfondir :

- Notre connaissance du problème de la mobilité des personnes avec déficiences visuelles (quelle information spatiale requièrent-elles ? comment cette information doit-elle être traitée et présentée? sous quelle modalité sensorielle ?...).

- Notre connaissance des calculs effectués par notre cerveau pour bien interpréter -en vu d'un déplacement- les signaux de l'environnement.

\section{Remerciements}

Bourse franco-mexicaine SFERE-CONACYT.

\section{BIBLIOGRAPHIE}

[Bac69] Bach-y-Rita P., Collins, C., Saunders, F., White, F., and Scadden, L. Vision substitution by tactile image projection. Nature, Vol. 221, No 963 (1969).

[Ben02] Benali Khoudja, M. Une Etude Physiologique et un Etat de l'Art Technologique sur le Rendu Tactile, Rapport CEA No : 02RT.016/Rev.0, France (2002).

[Ben73] Benjamin, J., Ali, N., Schepis, A. A Laser Cane for the Blind, Proc. of San Diego Biomedical Symposium, Vol. 12 (1973) 53-57.

[Ber97] Berthoz, A., Le sens du mouvement, Odile Jacob, 1997.

[Bis80] Bissit, D., Heyes, D. An Application of Biofeedback in the Rehabilitation of the Blind. Applied Ergonomics, Vol.11, No.1 (1980) 31-33.

[Bli70] Bliss, J. Dynamic Tactile Displays in ManMachine Systems. IEEE Transactions on Man-Machine Systems. (Special issue: Tactile displays conference), Vol. 11, No 1 (1970).

[Bor90] Borenstein, J. The NavBelt - A Computerized Multi-Sensor Travel Aid for Active Guidance of the Blind. Proc. of the $5^{\text {th }}$ Annual Conference on Technology and 
Persons with Visual Disabilities, Los Angeles, USA (1990) 107-116.

[Far02] Farcy, R., Damaschini, R., Leroux, R. Autonomic - Workshop \& Exhibition, Paris, (2002).

[Fu87] Fu, K., Gonzales, R., Lee, C. Robotics: Control, Sensing, Vision and Intelligence. McGraw Hill, New York (1987).

[How93] Howe, R., Kontarinis, D. Tactile Display of Contact Shape in Dexterous Telemanipulation. International Symposium on Haptic Interfaces for Virtual Environments and Teleoperator Systems, New Orleans, USA (1993).

[Kay74] Kay, L. A Sonar Aid to Enhance Spatial Perception of the Blind: Engineering Design and Evaluation. Radio and Electronic Engineer, Vol. 44, No. 11 (1974) 605-627.

[Led90] Lederman, S., Klatzy, R. Haptic classification of common objects: Knowledge-driven exploration. Cognitive Psychology, Vol. 22 (1990) 421-459.

[Mei98] Meijer, P. Cross-Modal Sensory Streams. International Conference on Computer Graphics and Interactive Techniques, Orlando, USA (1998) 184-192.

[Met] METEC GmbH 65a , D - 70176, Stuttgart, Germany http://www.metec-ag.de

[Mos92] Moss-Salentijn, L. The Human Tactile System. Nicholls, H. (ed.): Advanced Tactile Sensing for Robotics. Vol. 5 (1992) 123-150.

[Nfb] National Federation for the blind, updated information available at: http://nfb.org

[Oms02] World Health Organization. The World Health Report 2002 - Reducing Risks, Promoting Healthy Life. Geneva, Switzerland (2002).

http://www.who.int/whr/2002/

[Pis00] Pissaloux, E., Abdallah, S. Towards a Vision System for Blinds. International Symposium on Robotics Systems, Boston, USA (2000) 270-276.

[Pct00] Pissaloux, E., Le Coat, F., Tissot, A., Durbin, F. An adaptive parallel system dedicated to projective image matching, IEEE International Conference on Image Processing, Vancouver, Canada, (2000).

[Pis03] Pissaloux, E. Compensation de la déficience visuelle, Pruski, A., (ed.): Systèmes d'aides à l'handicap, Hermes (2003).

[Shi93] Shimoga, K. A Survey of Perceptual Feedback Issue in Dexterous Telemanipulation: Part II. Finger Touch Feedback. Proc. of Virtual Reality Annual International Symposium, Seattle, USA (1993) 271-279.

[Shi98] Shinohara, M., Shimizu, Y. Three-Dimensional Tactile Display for the Blind. IEEE Transactions on Rehabilitation Engineering, Vol. 6, No. 3 (1998) 249-256.

[Sri97] Srinivasan, M., Basdogan, C. Haptics in Virtual Environments: Taxonomy, Research Status, and Challenges. Computers and Graphics, Vol. 21, No. 4 (1997) 153-165.

[Tho00] Thorsten M., Karlheinz M. and Johannes S. The Heidelberg Tactile Vision Substitution System. 7th International Conference on Computer Helping People with Special Needs, Karlsruhe, Germany (2000) 256-262. [Ulr01] Ulrich, I., Borenstein, J. The Guide Cane Applying Mobile Robot Technologies to Assist the
Visually Impaired. IEEE Transactions on System, Man and Cybernetics - Part A: Systems and Humans, Vol. 31, No. 2 (2001) 131-136.

[Wor] Wormald International Sensory Aids, 6140 Horseshoe Bar Rd., Loomis, CA 95650, USA. 